\title{
Ultrasonic microwave-assisted extraction of antioxidant phenolics from waste jackfruit (Artocarpus heterophyllus Lam.) peels
}

\author{
Zhiguo JIANG ${ }^{1, a}$, Yanhua WANG ${ }^{2, b^{*}}$ \\ ${ }^{1}$ College of Food Science, Hainan University Haikou, China \\ ${ }^{2}$ Department of Applied Mathematics, School of Information Science and Technology, Hainan \\ University Haikou, China \\ ajzg6666@163.com, bwangyanhua0007@163.com
}

Keywords: Jackfruit peels ; Phenolics; Antioxidant; Ultrasonic microwave-assisted extraction; Response surface methodology

Abstract: An efficient extraction and purification technique based on ultrasonic microwave-assisted extraction (UMAE) method was established to obtain phenolics-enriched fraction from jackfruit byproducts (JPEF). Optimisation of the extraction parameters for maximum extraction yield of JPEF was carried out using response surface methodology (RSM). Results demonstrated that the optimum conditions were: ethanol concentration of $63 \%$, solvent-to-solid ratio of $34 \mathrm{~mL} / \mathrm{g}$, microwave power of $160 \mathrm{~W}$ and irradiation time of $20 \mathrm{~min}$. Under the optimal extraction conditions, the phenolics extraction yield was $8.14 \mathrm{mg}$ GAE/g DW. Compared to conventional extraction methods, UMAE provided higher extraction efficiency and activity. The results demonstrated that UMAE is a green method and have great potential for the extraction of active components and antioxidants from natural products.

\section{Introduction}

Jackfruit (Artocarpus heterophyllus) is the largest fruit in the world, and mainly cultivated in and around tropical areas. Jackfruit are rich in vitamins and minerals, and offers numerous health benefits [1,2]. After processing, jackfruit residues, e.g. peel, is usually discarded as a waste and prone to microbial spoilage, which was not only a waste of resource, but also pollution to the environment. In recent years, many polyphenolic compounds have exhibited various biological activities, including antioxidant [3], anti-inflammatory [4] and antimicrobial activities [5], and so forth. However, to the best of our knowledge, few information is available about the phenolics from jackfruit by-products (peels).

Extraction of phenolics is one of the most imperative steps for both application and further research and development. Various efficient and advanced extraction methods have been investigated, such as super critical fluid $\left(\mathrm{CO}_{2}\right)$ extraction (SCFE), ultrasound-assisted extraction (UAE), and microwave-assisted extraction (MAE). However, these extraction techniques have drawbacks to some extent. For example, despite representing non-organic solvents residing as advantage, SCFE equipment is expensive with a high-energy consumption [6]. Microwave-assisted extraction offers a rapid, clean, safe, and cost-effective method for heating that can cause a thermal effect by polarizing macromolecules, leading to alignment with electromagnetic field poles that may cause the breakage of hydrogen bonds, while its disadvantage is inhomogeneous heating [7,8]. Ultrasound-assisted extraction relies on acoustic cavitation, which causes disruption of cell walls of plant materials, resulting in a more extensive release of internal cell compounds and a more homogeneous system $[9,10]$. Thus, ultrasonic microwave-assisted extraction (UMAE) may be an effective complementary technology for dramatically accelerating the extracting process, improving selectivity and simplifying manipulation [11].

In the present study, UMAE technique was used as rapid and efficient extraction process for phenolics from jackfruit peels for high yields. Several processing parameters that could potentially influence the extraction efficiency were analyzed and optimized using RSM. 


\section{Experimental}

\section{Samples}

Fresh jackfruit fruits were purchased from local fruit center in Haikou City, Hainan Province of China. It was manually peeled and cut into small pieces, which were dried in a hot air oven at $60{ }^{\circ} \mathrm{C}$ for $48 \mathrm{~h}$ before being grinded and powdered (60 mesh).

\section{Chemicals}

ABTS [2,2'-azino-bis (3-ethylbenzthiazoline-6-sulfonic acid)] and Folin-Ciocalteu reagent were provided by Huadong Chemicals Co. (Hangzhou, China). Trolox, gallic acid, chlorogenic acid, catechin, rutin, quercetin, norartocarpetin and artocarpesin were obtained from Sigma Chemical Co. (St, Louis, MO, USA). Acetonitrile of HPLC grade was purchased from Merk (Darmstadt, Germany). The other chemical reagents were of analytical grade.

\section{Ultrasonic and microwave assisted extraction}

Phenolic compounds from powers of jackfruit (Artocarpusheterophyllus Lam.) peel were extracted using a domestic oven system (CW-2000, Shanghai Xin-tuo Microwave Instrument Co. Ltd., Shanghai, China). The apparatus was equipped with an open microwave with maximal power of $800 \mathrm{~W}$ at a frequency of $2450 \mathrm{MHz}$, and an ultrasonic transducer with a fixed power of $50 \mathrm{~W}$ at a frequency of $40 \mathrm{KHz}$. For each extraction, the pre-treated jackfruit peel powders $(1 \mathrm{~g})$ was weighed accurately and then transferred into the flask and proper volume (assigned according to the experimental design) of aqueous ethanol was added. Subsequently, the flask was transferred into the chamber of the apparatus connected with condensing tubes. Finally, the door of chamber was closed and the program of the parameters (microwave power and extraction time) according to the experiment planning was set for the extraction of phenolic compounds. When the extraction was accomplished, the flask was removed from apparatus and the resulting mixture was filtrated, then the volume of the filtrate and phenolic content in it were measured. Triplicate experiments were done for each design.

\section{Response surface methodology (RSM)}

Single-factor-test was employed to determination the preliminary range of the extraction variables including $X_{1}$ (ethanol concentration, \%), $X_{2}$ (microwave power, W), $X_{3}$ (irradiation time, min) and $X_{4}$ (solvent-to-solid ratio, $\mathrm{mL} / \mathrm{g}$ ). Then, a three-level-four-factor Box-Behnken design (BBD) was conducted to determine the best combination of extraction variables for maximum recovery of total phenolic compounds (TPC) from jackfruit peels. Regression analysis was performed on the basis of the experimental data and fitted to a quadratic polynomial model presented in the following equitation:

$\mathrm{Y}=\beta_{0}+\sum_{i=1}^{4} \beta_{i} X_{i}+\sum_{i=1}^{4} \beta_{i l} X_{i}^{2}+\sum_{i=1}^{3} \sum_{j=i+1}^{4} \beta_{i j} x_{i} x_{j}$

Where $Y$ is the predicted response (the yield of TPC in $\mathrm{mg}$ gallic acid equivalents (GAE)/g dry weight (DW) basis); $\beta_{0}, \beta_{i}, \beta_{i i}, \beta_{\mathrm{ij}}$ are the regression coefficients for intercept, linearity, square and interaction, respectively; $X_{i}$ and $X_{j}$ are the independent variables.

\section{Determination of total phenolic}

Total phenolics (TPs) content of the extract from jackfruit peels were determined using FolinCiocalteu reagent, according to a procedure described by Altemimi et al. [12].

\section{Total antioxidant capacity assay}

The total antioxidant capacity assay of the crude extract or fractions was determined with ABTS method, according to the procedure described by Mocan et al. [13], with slightly modification. 


\section{Results and discussion}

\section{Single factor experiment}

\section{Effect of ethanol concentration}

Extraction process was carried out at various ethanol concentration $(30 \%, 40 \%, 50 \%, 60 \%$ and $70 \%$ ), while other parameters were fixed and set as follows: microwave power $100 \mathrm{~W}$, extraction time $15 \mathrm{~min}$ and solvent-to-solid ratio $30 \mathrm{~mL} / \mathrm{g}$. The effect of ethanol concentration on extraction yield of phenolics was shown in Fig. 1A. The yield of phenolics increased first and then reduced with the further increase of ethanol concentration, and reached the maximum $(6.68 \mathrm{mg} \mathrm{GAE} / \mathrm{g})$ at $60 \%$ ethanol aqueous solution. It was reported that water is acted as the plant swelling agent, while ethanol is believed to disrupt the bonding between the target components and plant matrices $[14,15]$. Hence, the ethanol concentration of $60 \%$ was suitable for the extraction of phenolics.

\section{Effect of solvent-to-solid ratio}

In this study, the effect of ratio of solvent to solid on extraction yield of phenolics from jackfruit peels was investigated, and the results were listed in Fig. 1B. The ratio of solvent to solid was set at $10,20,30,40$, and $50 \mathrm{~mL} / \mathrm{g}$, while other extraction parameters were fixed as follows: ethanol concentration of $60 \%$, microwave power $100 \mathrm{~W}$ and irradiation time $15 \mathrm{~min}$. It could be founded that the extraction yield of phenolics continued to increase evidently with the increase of ratio of solvent to solid, and reached to a maximum value at $30 \mathrm{~mL} / \mathrm{g}$. However, as the ratio exceeded 30 $\mathrm{mL} / \mathrm{g}$, the extraction yields were almost constant regardless of increase of ratio of solvent to solid [16]. Considering the saving of solvent and easy operation, the ratio of $30 \mathrm{~mL} / \mathrm{g}$ was used as the following optimized experiments.

\section{Effect of microwave power}

The effects of microwave power on the yield of phenolics from jackfruit peel were investigated at levels ranging from 50 to $200 \mathrm{~W}$ with fixed ethanol concentration of $60 \%$, extraction time of $15 \mathrm{~min}$ and solvent-to-solid ratio of $30 \mathrm{~mL} / \mathrm{g}$. As shown in Fig. $1 \mathrm{C}$, the yield of phenolics increased with the increase of microwave power at the beginning of extraction, and reached the maximum (7.30 $\mathrm{mg} \mathrm{GAE} / \mathrm{g}$ ) at $150 \mathrm{~W}$. It could be due to the fact that microwave absorption causes fast internal heating, thus generating significantly high internal pressures in plant material. This causes the cell walls to swell and burst, thus promoting the release and dissolution of target components into the solvent [17,18]. Therefore, microwave power level $150 \mathrm{~W}$ was selected for further RSM optimization.

\section{Effect of irradiation time}

Generally speaking, by increasing the irradiation time, the quantity of target compounds extracted is increased, although there a risk of degradation of extracted active compounds. In the present study, the yield of phenolics was investigated at various irradiation times (5-25 min) with three other fixed factors: ethanol concentration of $60 \%$, microwave power $100 \mathrm{~W}$ and solvent-to-solid ratio of 30 $\mathrm{mL} / \mathrm{g}$. As shown in Fig. 1D, the first increase of irradiation time within 5-15 min resulted in a significant increase of phenolics yield, and then the further increase of irradiation time led to the decrease of phenolics yield beyond $15 \mathrm{~min}$. The findings indicated that prolonging irradiation time was not in favor of the yield of phenolics, possibly due to the chemical structural destruction and the decomposition of active compounds in longer exposure periods [19]. As a result, 15 min was an appropriate irradiation time for further UMAE experiments. 

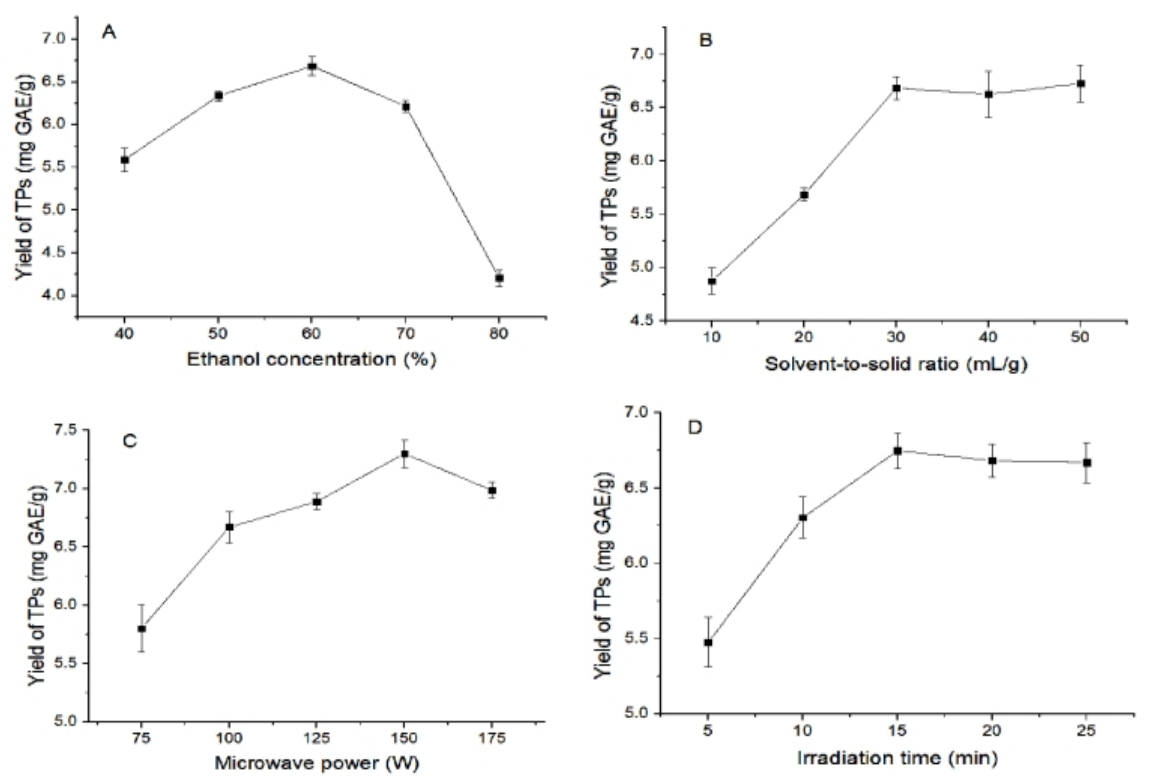

Fig. 1. Effect of different extraction factors on the yield of TPs from jackfruit peels

\section{Optimization of UMAE by RSM}

\section{Statistical analysis and the model fitting}

BBD with four factors and three levels were empolyed to optimize the mutual effect of four independent variables (ethanol concentration, solvent-to-solid ratio, microwave power and irradiation time) on the extraction yield of phenolics. Table 1 showed the design matrix and the extraction yields of phenolics. The response variable and the independent variables could be related using the following second-order equation:

$Y=7.83+0.90 X_{1}+0.46 X_{2}+0.23 X_{3}+0.056 X_{4}+0.090 X_{1} X_{2}-0.26 X_{1} X_{3}+0.42 X_{1} X_{4}+0.19 X_{2} X_{3}$ $+5.0 \times 5 .^{-3} X_{2} X_{4}+0.21 X_{3} X_{4}-2.13 X_{1}^{2}-0.65 X_{2}^{2}-0.53 X_{3}^{2}-0.067 X_{4}^{2}$

Statistical testing of the developed model was performed in the form of analysis of variance (ANOVA) by software of Design-Expert 8.0.5. The ANOVA for the experiment results are listed in Table 2. As shown in Table $3, F$-value $(F=24.45)$ and $p$-value $(p=0.0537)$ of the lack of fit implied that it was not significant relative to the pure error, which further confirmed that the fitted model adequately represented the experimental results under any combination of values of the variables during UMAE. The value of the determination coefficient $\left(R^{2}\right)$ evaluated from the secondorder regression model was 0.9719 , while the value of adjusted R-square $\left(R_{\text {adj }}^{2}\right)$ was 0.9437 , indicating a good degree of correlation between the actual and predicted values [20]. At the same time, the relatively low value of coefficient of variation $(\mathrm{C} . \mathrm{V} .=4.76 \%)$ demonstrated a high degree of precision and good reliability of the experimental results. All these results above suggested that the fitted model could work well for the prediction of TPC extract from jackfruit peels.

The $F$ and $p$-values are used as a tool to confirm the significance of each coefficient. The smaller the $P$-value, the more significant the corresponding coefficient [21]. According to the $p$ and $F$ values (Table 3 ), the linear coefficients $\left(X_{1}, X_{2}\right.$ and $\left.X_{3}\right)$, quadratic term coefficients $\left(X_{1}^{2}, X_{2}^{2}\right.$ and $\left.X_{3}^{2}\right)$ and cross product coefficients $\left(X_{1} X_{4}\right)$ were significant, with very small $P$-value $(p<0.05)$. Meanwhile, the ethanol concentration was the most significant factor affecting the extraction yield of phenolics. 
Table 1 Coded and non-coded Box-Behnken design with the experimental result.

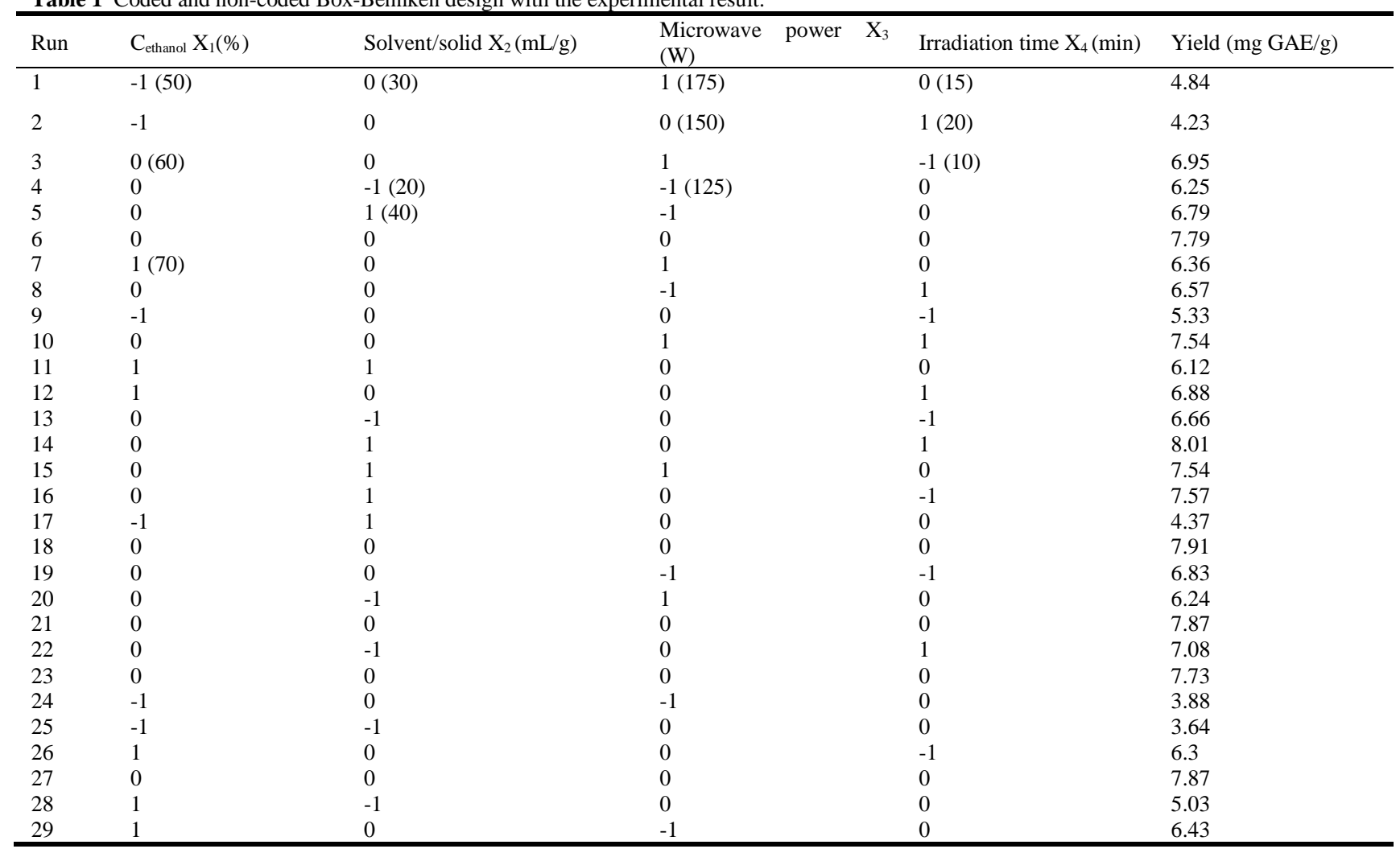

Table2 Analysis of variance for the fitted quadratic polynomial equation of extraction of phenolics.

\begin{tabular}{|c|c|c|c|c|c|}
\hline Source & Sum of squares & $\mathrm{df}$ & Mean square & $F$-value & $p$-value \\
\hline Model & 45.34 & 14 & 3.24 & 34.55 & $<0.0001 * *$ \\
\hline$X_{1}$ & 9.77 & 1 & 9.77 & 104.29 & $<0.0001 * *$ \\
\hline$X_{2}$ & 2.52 & 1 & 2.52 & 26.90 & $0.0001^{* *}$ \\
\hline$X_{3}$ & 0.62 & 1 & 0.62 & 6.58 & $0.0225^{*}$ \\
\hline$X_{4}$ & 0.037 & 1 & 0.037 & 0.40 & 0.5377 \\
\hline$X_{1} X_{2}$ & 0.032 & 1 & 0.032 & 0.35 & 0.5659 \\
\hline$X_{1} X_{3}$ & 0.27 & 1 & 0.27 & 2.83 & 0.1147 \\
\hline$X_{1} X_{4}$ & 0.71 & 1 & 0.71 & 7.53 & $0.0158^{*}$ \\
\hline$X_{2} X_{3}$ & 0.14 & 1 & 0.14 & 1.54 & 0.2349 \\
\hline$X_{2} X_{4}$ & 0.0001 & 1 & 0.0001 & 0.0011 & 0.9744 \\
\hline$X_{3} X_{4}$ & 0.18 & 1 & 0.18 & 1.93 & 0.1868 \\
\hline$X_{1}^{2}$ & 29.54 & 1 & 29.54 & 315.20 & $<0.0001 * *$ \\
\hline$X_{2}^{2}$ & 2.72 & 1 & 2.72 & 29.05 & $<0.0001 * *$ \\
\hline$X_{3}^{2}$ & 1.84 & 1 & 1.84 & 19.65 & $0.0006^{* *}$ \\
\hline$X_{4}^{2}$ & 0.029 & 1 & 0.029 & 0.31 & 0.5884 \\
\hline Residual & 1.31 & 14 & 0.094 & & \\
\hline Lack of Fit & 1.29 & 10 & 0.13 & 24.45 & 0.0537 \\
\hline Pure Error & 0.021 & 4 & 0.0053 & & \\
\hline Cor. Total & 46.65 & 28 & & & \\
\hline
\end{tabular}




\section{Analysis of reponse surface}

The relationship between test variables and total phenolics yield was visualized by response surface plots. Fig. 2A-C showed the ethanol concentration $\left(X_{1}\right)$ exerted significant effect on the extraction yield. An increase in the yields of TPs was observed with increasing ethanol concentration, but the trend was reserved when ethanol concentration reached beyond $60 \%$ and the extraction yield decreased thereafter. It can be seen that the interactions between ethanol concentration $\left(X_{1}\right)$ and irradiation time $\left(X_{4}\right)$ were significant $(p<0.05)$ because of the elliptical contour plots shown in Fig. $2 \mathrm{C}$, which was also confirmed by the results in Table 2. From Fig. 2D, both microwave power $\left(X_{3}\right)$ and solvent-to-solid ratio $\left(X_{2}\right)$ had quadratic effect on the yield of TPs. Extraction yield evidently increased at first and then declined quickly with the increase of the two parameters. Similar to Fig. $2 \mathrm{~A}$ and $\mathrm{B}$, solvent-to-solid ratio $\left(X_{2}\right)$ and microwave power $\left(X_{3}\right)$ was significant to the phenolics yield whereas irradiation time $\left(X_{4}\right)$ exerted a weaker effect on the yield of TPs, which was depicted in Fig. 2E and F.

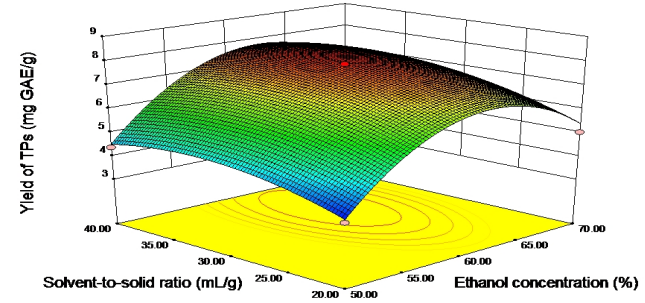

A

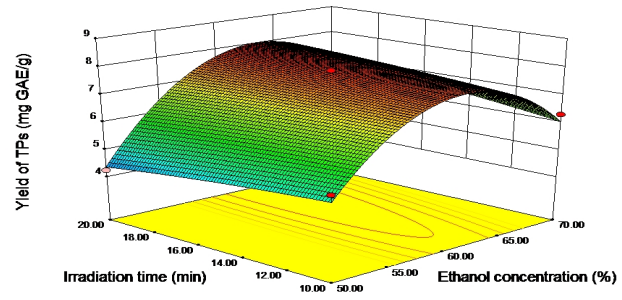

C

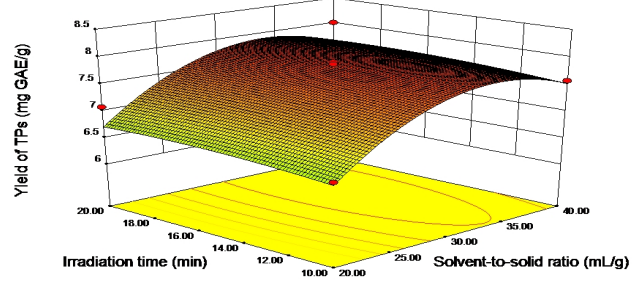

$E$

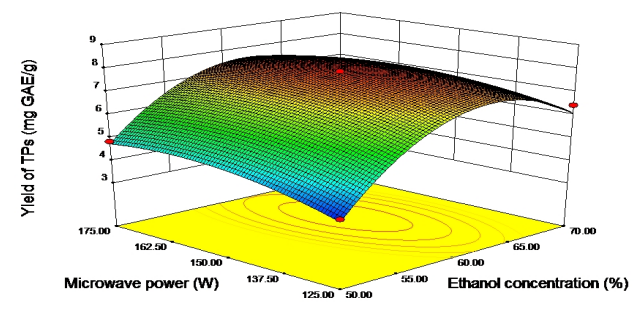

B

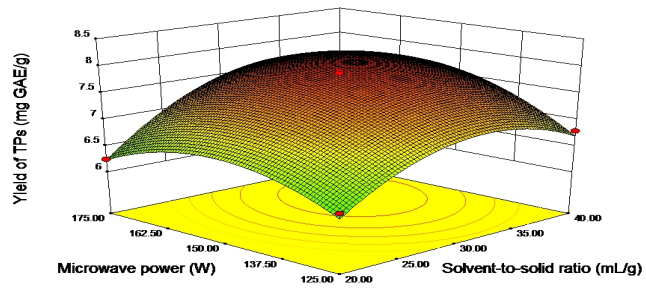

D

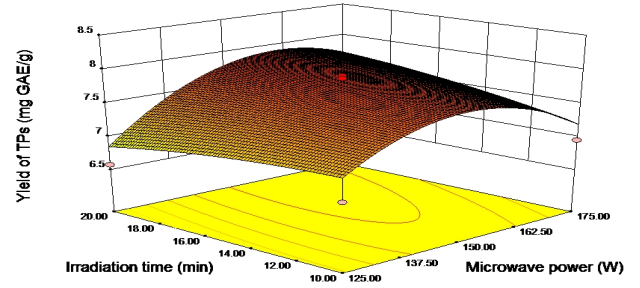

$\mathrm{F}$

Fig. 2. Response surface plots of from Box-Behnken design for the extraction of phenolics from jackfruit peels.

\section{Optimization of extracting parameters and validation of the model}

According to regression model, the optimum conditons were ethanol concentration $62.93 \%$, solvent-to-solid ratio $34.39 \mathrm{~mL} / \mathrm{g}$, microwave power $160.49 \mathrm{~W}$ and irradiation time $20 \mathrm{~min}$. Under above conditions, the model predicted a maximum response of $8.21 \mathrm{mg}$ GAE/g DW. Considering the actual operability, the optimized condition was modified as following: ethanol concentration $63 \%$, solvent-to-solid ratio of $34 \mathrm{~mL} / \mathrm{g}$, microwave power of $160 \mathrm{~W}$ and irradiation time $20 \mathrm{~min}$. To validate the suitability of the model equation, a verification experiment was carried out. In the verification experiment, the average yield of phenolics was $8.14 \mathrm{mg} \mathrm{GAE} / \mathrm{g} \mathrm{DW}(\mathrm{n}=3)$, which was no significant difference from the predicted value within the $95 \%$ confidence interval. This good correlation confirmed that the response model was adequate for reflecting the expected optimization. 


\section{Comparison of UMAE with HWE, MAE and UAE}

The yield and antioxidant capacity of phenolics obtained by UMAE was compared with traditional hot water extraction (HWE), ultrasound-assisted extraction (UAE) and microwave-assisted extraction (MAE), and the results were depicted in Table 3. Compared with other typical extraction methods, UMAE method could obviously improve the yield and antioxidant capacity of phenolic extracts by $13.76 \%-33.29 \%$ and $32.35 \%-51.38 \%$, respectively. The result indicated that the ultrasonic microwave-assisted technique possessed good synergistic effect of the acoustic cavitation and fast heating on extraction of antioxidant constituents from jackfruit peels. The above results suggested UMAE was an attractive and efficient sample preparation method and possessed good potential for the extraction of phenolics from jackfruit by-products.

\begin{tabular}{lll}
\multicolumn{2}{l}{ Table 3 Comparison of different extraction methods for phenolics from jackfruit peels $(\mathrm{n}=3)$} \\
\hline Methods & Yield of TPs (mg GAE/g) & ABTS (mg TE/g) \\
\hline UMAE & $8.14 \pm 0.23$ & $35.95 \pm 2.21$ \\
HWE & $5.43 \pm 0.13$ & $17.48 \pm 1.48$ \\
MAE & $6.72 \pm 0.31$ & $20.22 \pm 2.01$ \\
UAE & $7.02 \pm 0.17$ & $24.32 \pm 1.96$ \\
\hline
\end{tabular}

\section{Conclusions}

An efficient extraction and purification An efficient extraction and purification technique based on ultrasonic microwave-assisted extraction (UMAE) method was established to obtain phenolicsenriched fraction from jackfruit by-products (JPEF). Optimisation of the extraction parameters for maximum extraction yield of JPEF was carried out using response surface methodology (RSM). Results demonstrated that the optimum conditions were: ethanol concentration of $63 \%$, solvent-tosolid ratio of $34 \mathrm{~mL} / \mathrm{g}$, microwave power of $160 \mathrm{~W}$ and irradiation time of $20 \mathrm{~min}$. Under the optimal extraction conditions, the phenolics extraction yield was $8.14 \mathrm{mg}$ GAE/g DW. Compared to conventional extraction methods, UMAE provided higher extraction efficiency and activity. The results demonstrated that UMAE is a green method and have great potential for the extraction of active components and antioxidants from natural products.

\section{Acknowledgements}

The work was supported by Natural Science Foundation of Hainan Province of China (318MS013), the National Natural Science Foundation of China (37460420, 31760484), Scientific Research Project of Hainan Provincial Universities of China (Hnky2015ZD-3) and Hainan University Start-up Scientific Research Projects of China (kyqd1630).

\section{References}

[1] A.F.de Faria, V.V.de Rosso, A,Z.Mercadante. Plant Foods Hum Nutr 64 (2009): 108-115

[2] S.B. Swami, N.J. Thakor, P.M. Haldankar, S.B. Kalse. Compr Rev Food Sci F 11 (2012): $565-576$

[3] N. Balasundram, K. Sundram, S. Samman. Food Chem 99 (2006): 191-203

[4] C.L. Hsu, S.C. Fang, G.C. Yen. Food Funct 4 (2013): 1216-1222

[5] M.J. Alves, I.C.F.R. Ferreira, H.J.C. Froufe, R.M.V Abreu, A. Martins, M. Pintado. J Appl Microbiol 115 (2013): 346-357

[6] L.F. Zhang, Z.L. Liu. Ultrason Sonochem 15 (2008): 731-737

[7] Z.X. Lou, H.X. Wang, S. Zhu, S.W. Chen, M.Zhang, Z.P. Wang. Anal Chim Acta 716(2012): 28-33

[8] X. Liu, Y. Hu, D.F. Wei. Med Chem Res 23 (2014): 2360-2367

[9] P. Pongmalai, S. Devahastin, N. Chiewchan, S. Soponronnarit. Sep Purif Technol 144 (2015): 37-45

[10] T. Fan, J. Hu, L. Fu, L.J. Zhang. Carbohydr Polym 115 (2015): 701-706 
[11] X.H. Xiao, X.X. Si, X. Tong, G.K. Li. Phytochem Anal 23 (2012): 540-546

[12] A. Altemimi, R. Choudhary, D.G. Watson, D.A. Lightfoot. Ultrason Sonochem. 24 (2015): 247-255

[13] A. Mocan, L. Vlase, D.C. Vodnar, C. Bischin, D. Hanganu, A.M. Gheldiu, R. Oprean, R. Silaghi-Dumitrescu, G. Crisan. Molecules 19 (2014): 10056-10073

[14] Liu Y, S.L. Wei, M.C. Liao. Ind Crops Prod 49 (2013): 837-843

[15] F..Dahmoune, B..Nayak, K..Moussi, H..Remini, K..Madani. Food Chem 166 (2015): 585-595

[16] Y.F. Zou, X.F. Chen, W.Y. Yang, S. Liu. Carbohydr Polym 84.(2011): 503-508

[17] C.X. Lu, H.X. Wang, W.P. Lv, C.Y. Ma, P. Xu, J. Zhu, J. Xie, B. liu, Q.L. Zhou. Chromatographia 74 (2011): 139-144

[18] J. Ahmad, T. Langrish. J Food Eng 109 (2012): 162-174

[19] Y. Sun, W. Xu, W. Zhang, Q. Hu, X. Zeng. Sep Purif Technol 78 (2011): 311-320

[20] E. Firatligil-Durmus, O Evranuz. LWT-Food Sci Technol 43 (2010): 226-231

[21] D.M. Wu, T.T. Gao, H.S. Yang, Y.Z. Du, C. Li, L.X Wei, T.Y. Zhou, J.M. Lu, H.T. Bi. Ind Crops Prod 66 (2015): 229-238 